\title{
Current updates in management of relapsed/ refractory small cell lung cancer
}

\author{
Omar Abughanimeh'1, Vinicius Ernani ${ }^{1}$, Alissa Marr ${ }^{1}$, Apar Kishor Ganti ${ }^{1,2}$ \\ 'Division of Oncology and Hematology, Department of Internal Medicine, University of Nebraska Medical Center-Fred \& Pamela \\ Buffett Cancer Center, Omaha, NE 68198-6840, USA. \\ ${ }^{2}$ Division of Oncology and Hematology, VA Nebraska Western lowa Health Care System, Omaha, NE 68198-6840,USA.
}

Correspondence to: Dr. Omar Abughanimeh, Division of Oncology and Hematology, University of Nebraska Medical CenterFred \& Pamela Buffett Cancer Center, 986840 Nebraska Medical Center, Omaha, NE 68198-6840, USA.

E-mail: omar.abughanimeh@unmc.edu

How to cite this article: Abughanimeh O, Ernani V, Marr A, Ganti AK. Current updates in management of relapsed/refractory small cell lung cancer. J Cancer Metastasis Treat 2020;6:50. http://dx.doi.org/10.20517/2394-4722.2020.110

Received: 4 Oct 2020 First Decision: 12 Nov 2020 Revised: 23 Nov 2020 Accepted: 3 Dec 2020 Published: 18 Dec 2020

Academic Editor: Robert Kratzke Copy Editor: Monica Wang Production Editor: Jing Yu

\begin{abstract}
Small cell lung cancer (SCLC) is an aggressive subtype of neuroendocrine tumor. It is characterized by a rapid doubling time and early development of metastatic disease. Despite being responsive to initial chemotherapy, most of the patients will have relapse of the disease within a few months. The prognosis of SCLC is dismal with a 5-year survival rate of less than 5\%. For that reason, management of SCLC has been an active area of research. The utilization of immunotherapy has provided promising results in treatment of SCLC in the front-line setting. Therefore, utilization of immunotherapy and targeted therapy is being studied in the setting of relapsed/ refractory disease, and currently, different clinical trials are exploring new drugs and further options. In this review, we will explore the latest updates in management of relapsed/refractory SCLC.
\end{abstract}

Keywords: Small cell lung cancer, relapsed small cell lung cancer, chemotherapy, immunotherapy, targeted therapy

\section{INTRODUCTION}

Lung cancer is a major public health concern. In 2020, it is estimated that the United States (US) will have more than 200,000 new cases, making lung cancer the second most common malignancy and leading in cancer-related mortality in both genders ${ }^{[1]}$. Small cell lung cancer (SCLC) accounts for $\approx 20 \%$ of the total lung cancer cases globally ${ }^{[2]}$. In the US, SCLC accounts for $16 \%$ of new lung cancer cases ${ }^{[3]}$. SCLC is divided

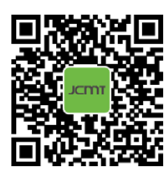


into limited-stage disease (LS-SCLC), which shows confined growth, or extensive-stage disease (ESSCLC) which is associated with metastasis. It is estimated that approximately $60 \%$ of SCLC patients present with extensive-stage disease at the time of initial diagnosis. The most common sites of spread include contralateral lung, adrenal glands, brain, liver, bones, and bone marrow ${ }^{[4,5]}$.

SCLC is highly responsive to chemotherapy ${ }^{[3,5]}$. The standard first-line treatment of LS-SCLC includes concurrent chemotherapy (cisplatin-etoposide) and radiation, while ES-SCLC is treated with a combination chemotherapy (platinum-etoposide) and an immune checkpoint inhibitor. For long time, the treatment of ES-SCLC consisted of platinum agents and etoposide ${ }^{[5]}$. This was changed recently due to results from IMpower-133 and CASPIAN studies, both of which demonstrated improved survival by adding atezolizumab and durvalumab, respectively, to platinum and etoposide ${ }^{[6,7]}$.

Despite being responsive to chemotherapy, most SCLC patients will experience tumor relapse within a few months, making management of these patients challenging ${ }^{[8,9]}$. If the relapse occurs within 3 months of treatment, the disease is called refractory or resistant, and the response to further treatment is $<10 \%{ }^{[9]}$. If the relapse occurs after 3 months, the expected response to further treatment is $25 \%$. Options for patients with refractory or relapsed disease are limited and patients with relapsed or refractory disease have a median survival of 8-9 months $^{[9,10]}$.

The treatment of refractory/relapsed SCLC has been an active area in research given the dismal prognosis and the poor outcome. Chemotherapy remains the cornerstone of treatment, but recently newer agents including immunotherapy are being studied, with promising results. In this review, we will discuss the current agents that are used in relapsed or refractory SCLC.

\section{TREATMENT OPTIONS OF RELAPSED/REFRACTORY SMALL CELL LUNG CANCER}

\section{Chemotherapy}

\section{Topotecan}

Topotecan is a semisynthetic water-soluble analog of camptothecin which acts as an inhibitor of the nuclear enzyme topoisomerase I, leading to DNA damage ${ }^{[11,12]}$. For a long time, it was the only drug that was approved by the Food and Drug Administration (FDA) for relapsed small cell lung cancer.

One of the earliest studies of topotecan in SCLC was conducted by the European Organization for Research and Treatment of Cancer ${ }^{[11]}$. This study was a phase II trial which included 92 patients (47 patients who were refractory to first-line treatment, and 45 patients had disease relapse after 3 months of stopping chemotherapy). In both arms, patients received intravenous (IV) topotecan at $1.5 \mathrm{mg} / \mathrm{m}^{2}$ for five consecutive days every 3 weeks. Topotecan demonstrated an overall response rate (ORR) of $6.4 \%$ (95\%CI: $1.3 \%-17.6 \%)$ in patients who failed first-line treatment, and 37.8\% (95\%CI: $23.8 \%-53.5 \%$ ) in patients who had disease relapse after 3 months of finishing chemotherapy treatment. The overall response in both groups was $21.7 \%$. The overall median duration of response was 7.6 months (95\%CI: 5.1-12.2 months), the median time to progression was 2.8 months (95\%CI: 2.2-3.9 months), and the overall survival (OS) was 5.4 months (95\%CI: 4.8-6.3 months). This study showed that topotecan had good activity in SCLC, specifically in patients who responded to initial chemotherapy ${ }^{[11]}$.

von Pawel et al. ${ }^{[12]}$ conducted a randomized phase III trial comparing topotecan to Cyclophosphamide, Doxorubicin, and Vincristine (CAV). In this study a total of 211 patients were recruited (107 treated with topotecan and 104 treated with CAV). Patients in the topotecan arm received IV topotecan $1.5 \mathrm{mg} / \mathrm{m}^{2}$ daily for five consecutive days every 3 weeks. The ORR of topotecan was $24.3 \%$ compared to $18.3 \%$ in the CAV arm. The median time to progression and median survival were similar in both arms (13.3 weeks vs. 12.3 weeks, and 25 weeks vs. 24.7 weeks, respectively). Despite the similarity in outcomes, this study showed that 
patients who received topotecan had better improvement in symptoms including dyspnea $(P=0.002)$, anorexia $(P=0.042)$, fatigue $(P=0.032)$, and hoarseness $(P=0.043)$.

Later in the early 2000s, a phase III clinical trial was conducted by O'Brien et al. ${ }^{[13]}$ to compare oral (PO) topotecan to supportive care alone. This study demonstrated prolonged survival with topotecan compared to supportive care. Moreover, it showed that patients who received topotecan had greater symptoms control and slower quality of life deterioration.

Eckardt et al. ${ }^{[14]}$ performed a randomized phase III clinical trial to compare PO topotecan with IV topotecan in relapsed SCLC. In this study, 309 patients were included, 153 patients received oral topotecan $2.3 \mathrm{mg} / \mathrm{m}^{2}$ daily for five consecutive days every three weeks whereas 151 received IV topotecan $1.5 \mathrm{mg} / \mathrm{m}^{2}$ daily for five consecutive days every three weeks. The study showed similar ORR in both arms of $18.3 \%$ vs. $21.9 \%$ respectively. There was no difference in median time to response (6.1 weeks for both), median duration of response (18.3 weeks vs. 25.4 weeks), and median time to progression (11.9 weeks vs. 14.6 weeks).

Hematologic complications have been commonly reported with topotecan. Despite having lower risk of grade 4 neutropenia compared to CAV, topotecan has higher risk of grade 4 thrombocytopenia and anemia $^{[12]}$. Non-hematologic toxicities were also reported such as fatigue, alopecia, nausea, and other gastrointestinal complications ${ }^{[11,12,14]}$.

Another study from Germany showed that a lower dose of topotecan $1.25 \mathrm{mg} / \mathrm{m}^{2}$ had a similar efficacy to the traditional dose of $1.5 \mathrm{mg} / \mathrm{m}^{2}$, but most importantly it was associated with reduced toxicity ${ }^{[15]}$. It is worth mentioning that there have been some studies which evaluated the usage of weekly topotecan instead of the standard regimen, however the results were not conclusive and weekly topotecan is no longer routinely used in clinical practice ${ }^{[9]}$.

\section{Lurbinectedin}

Lurbinectedin is a synthetic analog of trabectedin, which acts through inhibition of the active transcription protein-coding genes. This drug binds to CG-rich regions in the DNA causing irreversible arrest of elongating RNA polymerase on the DNA template, leading to accumulation of DNA breaks and apoptosis $^{[10,16]}$.

This drug was first studied in humans in 2009 when Elez et al. ${ }^{[17]}$ conducted a phase I clinical trial in patients with advanced solid tumors and showed both safety and anti-tumor effect. Later, lurbinectedin was studied in combination with doxorubicin in patients with relapsed SCLC. The study showed tolerability and an overall response rate of $57.7 \%{ }^{[18]}$.

Recently, a single arm, multicenter, phase II clinical trial was conducted on 105 patients who were treated with $3.2 \mathrm{mg} / \mathrm{m}^{2}$ of IV lurbinectedin every 3 weeks. Among them, 45 patient were considered to have resistant disease (defined as chemotherapy-free interval $<90$ days) and 60 patients with sensitive disease (defined as chemotherapy-free interval $\geq 90$ days). The study showed an ORR of 35.2\% (95\%CI: 26.2-45.2). Patients with sensitive disease had better ORR, [45\% (95\%CI: 32.1-58.4)] compared to the resistant disease group [22.2\% (95\%CI: 11.2-37.1)]. The overall median duration of response was 5.3 months (95\%CI: 4.16.4), which was also higher in the sensitive disease group 6.2 (95\%CI: 3.5-7.3) vs. 4.7 (95\%CI: 2.6-5.6). In this study, the median progression free survival (PFS) was 3.5 months (95\%CI: 2.6-4.3) and the median OS was 9.3 months (95\%CI: 6.3-11.8), both were also better in the sensitive disease group ${ }^{[16]}$. These results led to accelerated approval by the FDA to be used in patients with metastatic SCLC after progression on platinum-based chemotherapy. The National Comprehensive Cancer Network (NCCN) guidelines included lurbinectedin as a "preferred" agent in the second line treatment options along with topotecan or clinical trials ${ }^{[9]}$. 
Hematologic toxicities were reported with lurbinectedin treatment. Grade 4 neutropenia was recorded in $25 \%$ of the patients, whereas grade 4 thrombocytopenia was recorded in $4 \%$. None of the patients developed grade 4 anemia, however $9 \%$ of them developed grade 3 . Other non-hematologic toxicities included fatigue, decreased appetite and different gastrointestinal symptoms ${ }^{[16]}$.

Currently, a phase III clinical trial, the ATLANTIS study (NCT02566993) is being conducted to compare the activity of lurbinectedin combined with doxorubicin, with either topotecan or CAV as second line treatment for SCLC ${ }^{[10]}$.

\section{Irinotecan}

Irinotecan is a water-soluble derivative of camptothecin that acts through inhibition of DNA topotisomerase I, leading to antitumor effects ${ }^{[19]}$. The use of irinotecan in SCLC has been established in the last century. In 1990, a phase II study was conducted in Japan by Masuda et al. ${ }^{[19]}$. This study enrolled 16 patients with refractory or relapsed SCLC. All patients received IV irinotecan $100 \mathrm{mg} / \mathrm{m}^{2}$ every week. In this study, irinotecan led to an ORR of $47 \%$ (95\%CI: $21.4 \%-71.9 \%$ ). The median duration of response was 58 days (28-156 days). These findings were supported by another study that was done in Japan which demonstrated an ORR of 50\% (95\%CI: $25 \%-75 \%)^{[20]}$. A newer study was conducted in Japan which evaluated 30 patients with previously treated SCLC who received irinotecan $100 \mathrm{mg} / \mathrm{m}^{2}$ on days 1 , 8, every 3 weeks. The study showed an ORR of $41.3 \%$ (95\%CI: $25.5-59.3$ ) and a disease control rate of $69 \%$. The same study showed a median PFS and OS of 4.1 months and 10.4 months, respectively ${ }^{[21]}$.

The major toxicities associated with irinotecan treatment were hematologic, mostly leukopenia, followed by nausea and pulmonary toxicity ${ }^{[19]}$.

\section{Taxanes}

Paclitaxel was evaluated in multiple studies in patients with relapsed/refractory SCLC. A phase II trial was performed by Smit et al. ${ }^{[22]}$ in Netherlands where patients received IV paclitaxel $175 \mathrm{mg} / \mathrm{m}^{2}$ every 3 weeks. Paclitaxel led to an ORR of $29 \%$ (95\%CI: $12 \%-51 \%$ ). Furthermore, it was associated with a median duration of response of 108 days (64-243 days), median time to progression of 65 days (33-243days), and median survival of 100 days (23-262 days).

Paclitaxel was assessed in another phase II trial that was conducted by Yamamoto et al. ${ }^{[23]}$ who studied 21 patients with refractory SCLC. The study showed that single agent IV paclitaxel at a dose of $80 \mathrm{mg} / \mathrm{m}^{2}$ weekly had an ORR of $23.8 \%$ (95\% CI: 5.59-42.03), with a median survival of 5.8 months. The most common toxicity associated with paclitaxel was grade 3-4 neutropenia (66.6\%), other reported side effects included neuropathy, infections, and other gastrointestinal symptoms ${ }^{[23]}$.

Docetaxel is another taxane that was studied in relapsed/refractory SCLC. In the mid-1990s, docetaxel was studied in a phase II trial in patients with previously treated SCLC ${ }^{[24]}$. The study showed that IV docetaxel $100 \mathrm{mg} / \mathrm{m}^{2}$ once every 3 weeks was associated with an ORR of $25 \%$, with a median duration of response ranging between 3.5 and 12.6 months. The main toxicities reported in this trial were neutropenia, alopecia, and fatigue ${ }^{[24]}$.

It is worth to mention that Cabazitaxel was also studied in the setting of relapsed SCLC, but a study conducted by Evans et al. ${ }^{[25]}$ showed inferior PFS and OS when compared to topotecan.

\section{Temozolomide}

Temozolomide (TMZ) is an oral alkylating agent, which acts through production of O6 -alkyl-guanine lesions on DNA. These lesions are removed by $\mathrm{O} 6$-methylguanine-DNA methyltransferase (MGMT). 
However, if these lesions remain unrepaired, they can lead to cytotoxicity and ultimately apoptosis ${ }^{[26,27]}$. At its earliest stages, temozolomide was used in treating refractory astrocytoma and glioblastoma multiforme $e^{[26]}$.

In SCLC, several studies suggested that TMZ can be useful. Pietanza et al. ${ }^{[26]}$ performed a phase II study on 64 patients where 48 had sensitive disease (defined as having relapse or progression $>=60$ days after first line chemotherapy) and 16 had refractory disease. Patients received oral TMZ $75 \mathrm{mg} / \mathrm{m}^{2}$ daily for 21 days during a 28 -day cycle. The study reported an ORR of $23 \%$ (95\%CI: $12 \%-37 \%)$ in the sensitive group and 13\% (95\%CI: $2 \%-38 \%$ ) in the refractory group. Patients who had methylated MGMT experienced a higher response compared to patients with unmethylated MGMT ( $38 \%$ vs. $7 \%, P=0.08$ ). Interestingly, patients with brain metastasis had an ORR of $38 \%(95 \% \text { CI: } 14 \%-68 \%)^{[26]}$. Another study showed that TMZ can also be effective and tolerable using a regime of $200 \mathrm{mg} / \mathrm{m}^{2}$ daily for 5 days in 28-day cycles for patients with relapsed SCLC ${ }^{[28]}$. The most common toxicities reported with temozolomide were fatigue, gastrointestinal symptoms, and hematologic toxicities (most commonly lymphopenia $)^{[26]}$.

\section{Etoposide}

Etoposide has been used in treatment of SCLC for a long time. The use of etoposide in the second line setting has also been studied in patients who had received IV etoposide. A phase II trial showed that oral etoposide $50 \mathrm{mg} / \mathrm{m}^{2}$ daily for 21 days can lead to an ORR of $45.5 \%$ (95\%CI: $27 \%-65 \%$ ), median duration of response of 4 months (1.5-9.5 months), and median survival of 3.5 months (1-15 months) ${ }^{[29]}$. Another phase II trial showed a response rate of $23 \%{ }^{[30]}$. The most common observed toxicities were myelosuppression and alopecia $^{[29]}$.

\section{Vinorelbine}

Vinorelbine is a semisynthetic vinca alkaloid which acts through binding to microtubular proteins, preventing tubulin polymerization ${ }^{[31]}$. There are data that suggest efficacy of vinorelbine in the setting of SCLC. A phase II study was conducted on 26 patients with history of recurrent SCLC, who received vinorelbine $30 \mathrm{mg} / \mathrm{m}^{2}$ weekly, having shown a partial response rate of $16 \%$ (95\%CI: $4 \%-36 \%$ ) whereas $28 \%$ of the patients had stable disease ${ }^{[31]}$. Leukopenia was the major associated toxicity with vinorelbine as it occurred in $80 \%$ of the patients. Other common toxicities included anemia, gastrointestinal symptoms, and drug related fever ${ }^{[31]}$. Recently, a study in Poland showed that combining vinorelbine and cisplatin with electroporation (EP) was associated with increased anticancer activity due to the exposure of the cells to high intensity electric pulses, allowing the usage of lower doses of drugs ${ }^{[32]}$.

\section{Bendamustine}

Bendamustine is an alkylating agent that has been commonly used in different lymphoproliferative disorders. The clinical benefit of bendamustine in SCLC was demonstrated initially in a phase II clinical trial that was conducted in Europe. In this study, Schmittel and his colleagues ${ }^{[33]}$ enrolled 21 patients with SCLC who had a relapse $\geq 2$ months after completion of first line therapy. Twenty-one patients received bendamustine at a dose of $120 \mathrm{mg} / \mathrm{m}^{2}$ in the first two days every 3 weeks. The study showed a response rate of $29 \%$ with a median survival of 7 months.

Subsequently, another phase II study was conducted in North America for patients with relapsed SCLC where patients received $120 \mathrm{mg} / \mathrm{m}^{2}$ on the first 2 days of a 21 -day cycle. This study subdivided the population to a sensitive disease group; defined as stable or responsive disease to a platinum containing therapy for at least 90 days, or resistant disease group. A total of 50 patients participated with a response rate of $26 \%$ ( $95 \%$ CI: $13.3 \%-39.5 \%$ ). The response rate was higher in the sensitive disease group compared to the resistant disease group $(33 \%$ vs. $17 \%)$. The overall clinical benefit (complete response + partial response + stable disease) rate was $67 \%$. The median OS was 4.8 months (95\%CI: 3.8-6.3 months) which was also 
better in the sensitive group (5.7 months vs. 4.1 months) ${ }^{[34]}$. The most common toxicities were fatigue, anemia, thrombocytopenia, and different gastrointestinal symptoms ${ }^{[34]}$.

\section{Gemcitabine}

A phase II study showed that treatment with gemcitabine at a dose of $1000 \mathrm{mg} / \mathrm{m}^{2}$ on day 1 , day 8 and day 15 of a four-week cycle, resulted in an overall response rate of $13 \%$ (95\%CI: 6\%-27\%), with a median survival of 17 weeks (4-84 weeks) ${ }^{[35]}$. Interestingly, a different study tried gemcitabine $1,250 \mathrm{mg} / \mathrm{m}^{2}$ on day 1 and 8 every 3 weeks as a second line treatment of SCLC. But the results were not encouraging, as none of the 27 patients had a response ${ }^{[36]}$.

\section{Amrubicin}

Amrubicin is a 3rd generation anthracycline and a topoisomerase II inhibitor that has a chemical structure similar to doxorubicin ${ }^{[37,38]}$. The first data about amrubicin in SCLC was obtained from Japan after a phase II trial studied it in patients with untreated ES-SCLC. This study showed an overall response rate of $75.8 \%$ (95\%CI: $57.7 \%-88.9 \%$ ) and a median survival of 11.7 months (95\%CI: 9.9-15.3 months) ${ }^{[37]}$. Later, several studies were conducted, but the most notable one was a phase III clinical trial which compared amrubicin to topotecan in the second line setting. This trial evaluated 637 patients who were randomized 2:1 to amrubicin $40 \mathrm{mg} / \mathrm{m}^{2}$ daily for three days or topotecan $1.5 \mathrm{mg} / \mathrm{m}^{2}$ for 5 days every 3 weeks. Amrubicin was associated with a better overall response rate $(31.1 \% v s .16 .9 \%, P=0.001)$ and median PFS $(4.1$ months vs. 3.5 months, $P=0.01)$. However, it failed to show improvement in OS which was the primary endpoint ${ }^{[38]}$. This drug is not recommended in the NCCN guidelines for treatment of SCLC ${ }^{[9]}$.

\section{Vinflunine}

Vinflunine is a microtubule inhibitor that has been used in different malignancies including non-small cell lung cancer. Spigel and colleagues ${ }^{[39]}$ performed a phase II study on 51 patients with relapsed SCLC. Patients received IV vinflunine at $320 \mathrm{mg} / \mathrm{m}^{2}$ every 3 weeks. The study showed an ORR of $19.6 \% \%$ (95\%CI: 10-33\%). The median PFS and OS were 1.6 months (95\%CI: 1.3-3.9 months) and 4.9 months (95\%CI: 3.26.5 months) respectively. Despite being well tolerated, $5 \%$ of the patients had grade $3 / 4$ toxicities with neutropenia being the most common (32\%). Other side effects included fatigue (16\%), arthralgia (16\%), and different gastrointestinal symptoms ${ }^{[39]}$.

\section{Combined chemotherapy}

Multiagent chemotherapy have been the standard treatment for extensive-disease SCLC for long time ${ }^{[40,41]}$. Chemotherapy regimens like etoposide with platinum, CAV, and cyclophosphamide-doxorubicin-etoposide $(\mathrm{CDE})$ all showed reasonable response rate when used in the first line treatment. Nevertheless, the use of combined chemotherapy is limited in the setting of recurrent/relapsed SCLC, mainly due to intolerable toxicity at that stage ${ }^{[40]}$. The NCCN guidelines suggest only CAV in the 2 nd line setting ${ }^{[9]}$.

One of the few studies to evaluate combined chemotherapy agents in the 2nd line setting was a phase III trial from Japan which compared a combination of cisplatin-irinotecan-etoposide (CIE) to topotecan in the setting of relapsed SCLC. This study revealed that CIE had a better OS (18.2 months vs. 12.5 months; $P$ $=0.0079$ ). However, the toxicity was significantly higher in the CIE arm and therefore it is not commonly used in practice ${ }^{[42]}$.

Table 1 summarizes some of the clinical trials that evaluated different chemotherapy agents in relapsed/ refractory small cell lung cancer.

\section{Immunotherapy}

The utilization of the immune system in treating cancers has been an exciting field that is being developed over the last years. The immune system recognizes cancer cells but, in most situations, it is not able to 
Table 1. Clinical trials of chemotherapy agents in the setting of recurrent/relapsed SCLC

\begin{tabular}{|c|c|c|c|c|c|}
\hline Agent & Clinical trial design & $\begin{array}{c}\text { Median } \\
\text { response rate }\end{array}$ & $\begin{array}{c}\text { Median progression } \\
\text { free survival }\end{array}$ & $\begin{array}{l}\text { Median overall } \\
\text { survival }\end{array}$ & $\begin{array}{c}\text { Median duration } \\
\text { of response }\end{array}$ \\
\hline \multirow[t]{3}{*}{ Topotecan } & Phase III, IV topotecan vs. $\mathrm{CAV}^{[12]}$ & $24.3 \%$ & 13.3 weeks & 25 weeks & 14.4 weeks \\
\hline & Phase III PO topotecan vs. supportive care ${ }^{[13]}$ & $7 \%$ & 16.3 weeks & 25.9 weeks & Not reported \\
\hline & Phase III, IV vs. PO topoteca ${ }^{\text {n[14] }}$ & $\begin{array}{l}18.3 \%-\mathrm{PO} \\
21.9 \%-\mathrm{IV}\end{array}$ & $\begin{array}{l}11.9 \text { weeks -PO; } \\
14.6 \text { weeks - IV }\end{array}$ & $\begin{array}{l}33 \text { weeks - PO; } \\
35 \text { weeks - IV }\end{array}$ & $\begin{array}{l}18.3 \text { weeks - PO } \\
25.4 \text { weeks - IV }\end{array}$ \\
\hline Lurbinectedin & Phase II, single arm 2nd line ${ }^{[16]}$ & $35.2 \%$ & 3.5 months & 9.3 months & 5.3 months \\
\hline \multirow[t]{2}{*}{ Irinotecan } & Phase II, single arm 2nd line ${ }^{[19]}$ & $47 \%$ & Not reported & 187 days & 58 days \\
\hline & Phase II, single arm 2nd line ${ }^{[21]}$ & $41.3 \%$ & 4.1 months & 10.4 months & Not reported \\
\hline \multirow[t]{2}{*}{ Paclitaxel } & Phase II, single arm $\geq 2$ nd line ${ }^{[22]}$ & $29 \%$ & 65 days & 100 days & 108 days \\
\hline & Phase II, single arm $\geq 2$ nd line ${ }^{[23]}$ & $23.8 \%$ & Not reported & 5.8 months & Not reported \\
\hline Docetaxel & Phase II, single arm ${ }^{[24]}$ & $25 \%$ & 4.7 months & Not reported & 4.7 months \\
\hline Temozolomide & Phase II, single arm 2nd or 3rd line $e^{[26]}$ & $\begin{array}{l}23 \% \text { - sensitive } \\
13 \% \text { - refractory }\end{array}$ & 1.6 months & 5.8 months & 3.5 months \\
\hline \multirow[t]{2}{*}{ Etoposide } & Phase II, PO etoposide $\geq 2$ nd line ${ }^{[29]}$ & $45.5 \%$ & 4 months & $3.5+$ months & 4 months \\
\hline & $\begin{array}{l}\text { Phase II, single arm PO etoposide } \geq 2 \text { nd } \\
\text { line }^{[30]}\end{array}$ & $23 \%$ & Not reported & 18 weeks & $\begin{array}{l}C R=16 \text { weeks } \\
P R=9 \text { weeks }\end{array}$ \\
\hline Vinorelbine & Phase II, single arm 2nd line ${ }^{[31]}$ & $\begin{array}{l}P R=16 \% \\
S D=28 \%\end{array}$ & Not reported & Not reported & Not reported \\
\hline Bendamustine & Phase II, single arm 2nd and 3rd line ${ }^{[34]}$ & $26 \%$ & 4 months & 4.8 months & Not reported \\
\hline Gemcitabine & Phase II, single arm $\geq 2$ nd line ${ }^{[35]}$ & $13 \%$ & 8 weeks & 17 weeks & 10-20 weeks \\
\hline Amrubicin & Phase III, Amrubicin vs. topotecan 2nd line ${ }^{[38]}$ & $31.1 \%$ & 4.1 months & 7.5 months & 4.8 months \\
\hline Vinflunine & Phase II, single arm 2nd line ${ }^{[39]}$ & $19.6 \%$ & 1.6 months & 4.9 months & 2.7 months \\
\hline $\mathrm{CIE}$ & Phase III, CIE vs. topotecan 2nd line ${ }^{[42]}$ & $84 \%$ & 5.7 months & 18.2 months & Not reported \\
\hline CAV & Phase III, IV topotecan vs. CAV 2nd line ${ }^{[12]}$ & $18.3 \%$ & 12.3 weeks & 24.7 weeks & 15.3 weeks \\
\hline
\end{tabular}

SCLC: small cell lung cancer; CAV: Cyclophosphamide, Doxorubicin, and Vincristine; PO: compare oral; CIE: cisplatin-irinotecanetoposide; PR: partial response; SD: stable disease; CR: complete response

eliminate the cancer cells due to inhibitory receptors and signals (checkpoints). Programmed death-1 (PD-1) and cytotoxic lymphocyte antigen 4 (CTLA-4) are the most common checkpoints that have been studied in solid malignancies ${ }^{[8]}$. While immunotherapy is now recommended in the front-line setting, there have been trials in immunotherapy naïve patients with relapsed SCLC, that were conducted prior to availibility of IMPower 133 and CASPIAN results.

\section{Nivolumab}

The CheckMate 032 trial $^{[43]}$ evaluated nivolumab in the setting of recurrent SCLC. In this study, patients were randomized to three groups where they received either nivolumab $3 \mathrm{mg} / \mathrm{kg}$ alone every 2 weeks until disease progression, nivolumab $1 \mathrm{mg} / \mathrm{kg}+$ ipilimumab $3 \mathrm{mg} / \mathrm{kg}$ every 3 weeks for 4 cycles followed by maintenance nivolumab every 2 weeks, and nivolumab $3 \mathrm{mg} / \mathrm{kg}$ plus ipilimumab $1 \mathrm{mg} / \mathrm{kg}$ every 3 weeks for 4 cycles followed by maintenance nivolumab every 2 weeks. The number of patients in each group was 98,61 , and 54 respectively. A fourth group included only three patients who received nivolumab $1 \mathrm{mg} / \mathrm{kg}+$ ipilimumab $1 \mathrm{mg} / \mathrm{kg}$. The study showed a response rate of $10 \%$ for nivolumab alone, $23 \%$ for the nivolumab $1 \mathrm{mg} / \mathrm{kg}+$ ipilimumab $3 \mathrm{mg} / \mathrm{kg}, 19 \%$ for the nivolumab $3 \mathrm{mg} / \mathrm{kg}+$ ipilimumab $1 \mathrm{mg} / \mathrm{kg}$, and $33 \%$ for the nivolumab $1 \mathrm{mg} / \mathrm{kg}$ plus ipilimumab $1 \mathrm{mg} / \mathrm{kg}$. Interestingly the expression of programmed death-1 ligand (PD-L1) did not correlate with the response to therapy. Grade 3-4 treatment related toxicities were most common in the nivolumab $1 \mathrm{mg} / \mathrm{kg}$ + ipilimumab $3 \mathrm{mg} / \mathrm{kg}$ group (30\%) with diarrhea being the most common ${ }^{[43]}$. An updated analysis of the Checkmate 032 trial showed a higher response rate in the combination of nivolumab $1 \mathrm{mg} / \mathrm{kg}$ plus ipilimumab $3 \mathrm{mg} / \mathrm{kg}$ compared to nivolumab alone $(21.9 \% v s$. $11.6 \%$; odds ratio $2.12 ; 95 \%$ CI: $1.06-4.26, P$-value $=0.03)^{[44]}$. However, it demonstrated similar OS between the 2 groups. The median OS in the nivolumab group was 5.7 months (95\%CI: 3.8-7.6 months) compared to 4.7 months in the combination arm (95\%CI: 3.1-8.3 months). Furthermore, toxicities were higher in the combination arm. The last 2 findings led the NCCN panel to recommend nivolumab alone instead of the combination $^{[9]}$. 
The CheckMate 331 trial (NCT02481830) ${ }^{[45]}$ is an ongoing phase III clinical trial that is comparing nivolumab to topotecan and amrubicin. The trial estimated complete date is in mid-2021, however, preliminary data showed no significant difference in overall survival between nivolumab (median of 7.5 months) and chemotherapy (median of 8.4 months) with a hazard ratio of 0.86 (95\%CI: $0.72-1.04$ ).

\section{Pembrolizumab}

The KEYNOTE-028 trial (NCT02054806) ${ }^{[46]}$, is a phase Ib study that evaluated the safety of pembrolizumab $10 \mathrm{mg} / \mathrm{kg}$ every 2 weeks in patients with advanced PD-L1 positive ES-SCLC. This study revealed a promising efficacy of pembrolizumab in SCLC with an ORR of 33\% (95\%CI: 16\%-55\% $)^{[46]}$. The KEYNOTE-158 trial (NCT02628067 ${ }^{[47]}$, is an ongoing phase II trial, to evaluate the benefit of pembrolizumab in advanced SCLC. The preliminary results showed an ORR of $18.7 \%$ (95\%CI: $11.8 \%-27.4 \%)$. The response was higher in patients who had PD-L1 positive tumor compared to $\mathrm{PD}-\mathrm{L} 1$ negative tumor $(35.7 \% v s .6 \%)^{[47]}$. A recent paper was published by Chung et al ${ }^{[48]}$ who performed a combined analysis of both KEYNOTE-028 and KEYNOTE-158. The results demonstrated an ORR of 19.3\% (95\%CI: 11.4\%-29.4\%) with a median OS of 7.7 months (95\%CI: 5.2-10.1 months). As revealed by the KEYNOTE-158 trial, patients who had PD-L1 positive tumor had better ORR and OS. Nevertheless, the NCCN panel added pembrolizumab as a second line therapy regardless of the $\mathrm{PD}-\mathrm{L} 1$ results ${ }^{[9]}$.

\section{Durvalumab}

Durvalumab is another immunotherapy agent that was approved by the FDA in 2020 to be used with combined chemotherapy in the first line setting based on the CASPIAN trial ${ }^{[7]}$. A phase I study evaluated the use of durvalumab and tremelimumab in patients who had disease progression on at least one treatment. The results of this study showed an ORR of 13.3\%, PFS of 1.8 months and an OS of 7.9 months ${ }^{[49]}$. However, a phase II study did not show sufficient response of durvalumab and tremelimumab when it was used with or without radiation ${ }^{[50]}$.

\section{Atezolizumab}

Atezolizumab was approved by the FDA to be used in the front line setting based on the IMpower-133 trial which demonstrated significant improvement in the PFS and OS by adding atezolizumab to chemotherapy ${ }^{[2]}$. However, the use of it in the second line setting is still under study. A recent phase I trial on 17 patients showed that atezolizumab was tolerated and it had some efficacy with a median OS of 5.9 months $^{[51]}$.

There are no doubts that the field of immunotherapy will continue to expand, with many different clinical trials curently ongoing. Table 2 summarizes some of the trials that investigated immunotherapy in relapsed small cell lung cancer based on reported studies in literature.

\section{Targeted therapy}

Targeted therapy has been an exciting field for different malignancies. Until recently, its use in SCLC has not been successful ${ }^{[8]}$. Several trials were done to assess targeted therapies as a single agent or in combination with chemotherapy but many of them did not reach their primary endpoint ${ }^{[8]}$. These therapies included bevacizumab, vandetanib, aflibercept, vismodegib, cixutumumab, panobinostat, oblimersen, and obatoclax $^{[52-58]}$. However, there are some targeted therapies that have shown some promising results.

\section{Alisertib}

Alisertib is an oral aurora kinase A inhibitor ${ }^{[8]}$. Melichar et al. ${ }^{[59]}$ performed a study to evaluate alisertib use in different relapsed solid malignancies including SCLC. A total of 48 patients with SCLC were enrolled, with a total overall response rate of $21 \%$. However, the time to progression was only 2.6 months. 
Table 2. Clinical trials of immunotherapy in the setting of recurrent/relapsed SCLC

\begin{tabular}{|c|c|c|c|c|c|}
\hline Agent & Study design & $\begin{array}{c}\text { Overall } \\
\text { response }\end{array}$ & $\begin{array}{l}\text { Progression free } \\
\text { survival }\end{array}$ & Overall survival & $\begin{array}{c}\text { Median duration } \\
\text { of response }\end{array}$ \\
\hline \multirow[t]{4}{*}{ Nivolumab } & CheckMate-032: phase I/II & G1: $10 \%$ & G1: 1.4 months & G1: 4.4 months & G1 = not reached \\
\hline & Nivolumab 3mg/kg (G1) vs. Nivolumab 1mg/kg + & $\mathrm{G} 2: 23 \%$ & G2: 2.6 months & G2: 7.7 months & $\mathrm{G} 2=7.7$ months \\
\hline & $\begin{array}{l}\text { Ipilimumab 3mg/kg (G2) vs. Nivolumab 3mg/kg + } \\
\text { Ipilimumab 1mg/kg (G3) as } \geq 2 \text { nd line }{ }^{[43]}\end{array}$ & G3: $19 \%$ & G3: 1.4 months & G3: 6 months & $\mathrm{G} 3=4.4$ months \\
\hline & $\begin{array}{l}\text { CheckMate 331: phase III Nivolumab vs. } \\
\text { chemotherapy as } 2 \text { nd line }{ }^{[45]}\end{array}$ & $14 \%$ & 1.4 months & 7.5 months & 8.3 months \\
\hline \multirow{2}{*}{ Pembrolizumab } & KEYNOTE-028: phase Ib, $\geq 2$ nd line ${ }^{[46]}$ & $33.3 \%$ & 1.9 months & 9.7 months & 19.4 months \\
\hline & KEYNOTE-158: Phase II, $2^{\text {nd }}$ line ${ }^{[47]}$ & $18.7 \%$ & 2 months & 9.1 months & Not reached \\
\hline Durvalumab & Phase I, durvalumab + tremelimumab as $\geq 2$ nd line ${ }^{[49]}$ & $13.3 \%$ & 1.8 months & 7.9 months & 18.9 months \\
\hline
\end{tabular}

SCLC: small cell lung cancer

Veliparib

Veliparib is an inhibitor of poly (ADP-ribose) polymerase (PARP) ${ }^{[60]}$. A study compared the combination of veliparib with TMZ to TMZ and placebo in patients with relapsed/refractory SCLC. The study failed to show difference in PFS or OS, but it showed that the combination of TMZ and veliparib was associated with better ORR compared to the other group ( $39 \% v s .14 \%$ respectively, $P=0.016)^{[27]}$.

\section{Pazopanib}

Pazopanib is a tyrosine kinase inhibitor (TKI) of vascular endothelial growth factor receptors (VEGFR-1, VEGFR-2, and VEGFR-3), platelet-derived growth factor receptors (PDGFR), and c-kit. It showed promising results when used in the second line setting in refractory/relapsed SCLC ${ }^{[61]}$. In a phase II study, 39 patients with platinum sensitive disease and 19 patients with refractory disease received pazopanib $800 \mathrm{mg}$ daily. The partial response rate was $13.8 \%$ (95\%CI: 5-22.7), with $34.5 \%$ achieving stable disease. The median PFS was 2.5 months (95\%CI: 1.9-3.1 months) and OS was 6 months (95\%CI: 3.8-8.2 months). Interestingly, the study showed that one cycle of pazopanib resulted in significant decrease in number of patients with $\geq 5$ circulating tumor cells (CTCs) $/ 7.5 \mathrm{ml}$ blood. That led the authors to suggest consideration of CTCs enumeration as biomarker of response ${ }^{[6]]}$.

\section{Anlotinib}

Anlotinib is another TKI that targets VEGFR-2, VEGFR-3, PDGFR-b, and c-Kit. The "ALTER 1202" trial, is a phase II, double-blinded, randomized, placebo-controlled study that enrolled 120 patients with SCLC who had disease progression after at least 2 lines of treatment. Eighty-two patients received anlotinib $12 \mathrm{mg}$ daily for 2 weeks on and one week off cycle while the rest got placebo. The study demonstrated a significant improvement in PFS in the anlotinib arm [4.1 months (95\%CI: 2.8 to 4.2 months] compared to placebo [0.7 months (95\%CI: 0.7 to 0.8 months)] ( $P$-value $<0.0001)$. The disease control rate (DCR) was significantly higher in the anlotinib group compared to placebo $(71.6 \% v s .13 .2 \%, P \text {-value }<0.0001)^{[62]}$. Later, an update was published which also showed an improvement in OS in the anlotinib arm (7.3 months vs. 4.9 months) ${ }^{[63]}$.

Table 3 summarizes some of the clinical trials that involved targeted therapy in recurrent/relapsed SCLC.

\section{FUTURE CONSIDERATIONS AND CHALLENGES}

The treatment of relapsed/refractory SCLC has been challenging over the last several years given the lack of effective therapies. Till 2020, topotecan was the only FDA approved drug for relapsed SCLC before the FDA granted accelerated approval for lurbinectedin. There is no doubt that the management of SCLC is actively developing. Currently, there has been a focus on immunotherapy and targeted therapies in the relapsed/ refractory disease setting especially after the results of the IMpower 133 and CASPIAN trials. 
Table 3. Clinical trials of targeted therapies in the setting of recurrent/relapsed SCLC

\begin{tabular}{|c|c|c|c|c|c|}
\hline Agent & Study design & Overall response & $\begin{array}{c}\text { Progression free } \\
\text { survival }\end{array}$ & Overall survival & $\begin{array}{c}\text { Median duration of } \\
\text { response }\end{array}$ \\
\hline Alisertib & $\begin{array}{l}\text { Phase II, single arm } 2 \text { nd or } 3 r d \text { line in } \\
\text { different solid malignancies including } \\
\text { SCLC }\end{array}$ & $21 \%$ & 2.1 months & Not reported & 4.1 months \\
\hline Veliparib & $\begin{array}{l}\text { Phase II, veliparib + TMZ to TMZ + } \\
\text { placebo } 2 \text { nd line }{ }^{[27]} \text {. }\end{array}$ & $\begin{array}{l}39 \% \text { vs. } 14(P \text {-value }= \\
0.016)\end{array}$ & $\begin{array}{l}3.8 \text { months vs. } 2 \\
\text { months }(P=0.39)\end{array}$ & $\begin{array}{l}8.2 \text { months vs. } 7 \\
\text { months }(P=0.5)\end{array}$ & $\begin{array}{l}4.61 \text { months vs. } 3.68 \\
\text { months }(P=0.0507)\end{array}$ \\
\hline Pazopanib & Phase II, single arm 2nd line ${ }^{[61]}$ & $13.8 \% \mathrm{PR}$ & 2.5 months & 6 months & Not reported \\
\hline Anlotinib & $\begin{array}{l}\text { Phase II anlotinib vs. placebo as } \geq \\
\text { 3rd line }{ }^{[62,63]} \text {. }\end{array}$ & $\begin{array}{l}\text { DCR: } 71.6 \% \text { vs. } 13.2 \% \\
(P \text {-value }<0.0001)\end{array}$ & $\begin{array}{l}4.1 \text { months vs. } 0.7 \\
\text { months }\end{array}$ & $\begin{array}{l}7.3 \text { months vs. } 4.9 \\
\text { months }\end{array}$ & Not reported \\
\hline
\end{tabular}

SCLC: small cell lung cancer; TMZ: Temozolomide; DCR: disease control rate; PR: partial response

Table 4. Active clinical trials evaluating new treatments for recurrent/relapsed SCLC

\begin{tabular}{|c|c|c|c|}
\hline Study name/ClinicalTrials.gov ID & Study design & $\begin{array}{l}\text { Treatment arms } \\
\end{array}$ & Primary outcome \\
\hline ATLANTIS/NCT02566993 & Phase III & Lurbinectedin + doxorubicin vs. CAV vs. topotecan & Overall survival \\
\hline CheckMate 331/NCT02481830 & Phase III & Nivolumab vs. topotecan vs. amrubicin as 2 nd line & Overall survival \\
\hline TAHOE/NCT03061812 & Phase III & Rovalpituzumab vs. topotecan as 2 nd line & Overall survival \\
\hline KEYNOTE-158/NCT02628067 & Phase II & Pembrolizumab as 2 nd line & Overall response rate \\
\hline MCC-19163/NCT03406715 & Phase II & $\begin{array}{l}\text { Single arm: Ipilimumab + Nivolumab + Dendritic } \\
\text { Cell Based p } 53 \text { Vaccine as a 2nd line and beyond }\end{array}$ & Disease control rate \\
\hline AFT-17/NCT02963090 & Phase II & Topotecan IV vs. Pembrolizumab as 2nd line & Progression free survival \\
\hline CA001-030/NCT02247349 & Phase I/II & $\begin{array}{l}\text { BMS-986012 + nivolumab vs. BMS-986012 alone } \\
\text { as 2nd line }\end{array}$ & $\begin{array}{l}\text { Safety as measured by } \\
\text { frequency of adverse events. }\end{array}$ \\
\hline KEYNOTE-028/NCT02054806 & Phase I & Pembrolizumab as 2 nd line in different solid tumors & Overall response rate \\
\hline MEDIOLA/NCTO2734004 & Phase I/II & $\begin{array}{l}\text { Olaparib + bevacizumab + durvalumab vs. Olaparib } \\
+ \text { durvalumab as } 2 \text { nd line and beyond }\end{array}$ & $\begin{array}{l}\text { Disease control rate, safety and } \\
\text { tolerability of the drugs, overall } \\
\text { response rate. }\end{array}$ \\
\hline
\end{tabular}

SCLC: small cell lung cancer; CAV: Cyclophosphamide, Doxorubicin, and Vincristine

Table 4 summarizes some of the ongoing clinical trials to investigate new approaches for relapsed/refractory SCLC.

\section{EFFECT OF COVID-19 ON SCLC TREATMENT}

In late 2019, multiple cases of atypical pneumonia had been reported in Wuhan, China, caused by a novel type of coronavirus named severe acute respiratory syndrome coronavirus 2 (SARS-CoV-2), that led to the COVID-19 disease ${ }^{[64]}$. So far, its impact on lung cancer diagnosis and treatment is not well reported. However, a recent study from Spain did show that patients with lung cancer and COVID-19 infection have a higher mortality rate compared to the general population with COIVD-19 alone ${ }^{[61]}$. While there is a concern that treating these patients may increase the risk of complications associated with the SRA-CoV-2, relapsed SCLC has a very aggressive course. We recommend continuing treatment of these patients while monitoring for the development of COVID-19 disease.

\section{CONCLUSION}

In conclusion, relapsed SCLC remains a difficult disease with a dismal prognosis. Most of the patients will have disease relapse after a few months of first-line treatment. Till date, there are only 2 drugs approved by the FDA, topotecan and lurbinectedin both with modest efficacy. However, the recent advances in immunotherapy and targeted therapy are exciting, and the results of ongoing trials may help find a strategy that will improve outcomes for these patients.

\section{DECLARATIONS}

\section{Authors' contributions}

Wrote the manuscript: Abughanimeh O 
Reviewed, edited, and approved the final manuscript: Ernani V, Marr A, Ganti AK

Contributed to the manuscript: Abughanimeh O, Ernani V, Marr A, Ganti AK

\section{Availability of data and materials}

Not applicable.

\section{Financial support and sponsorship}

All authors have declared that they have no financial relationships at present that is related to this paper.

\section{Conflicts of interest}

All authors declared that there are no conflicts of interest.

\section{Ethical approval and consent to participate}

Not applicable.

\section{Consent for publication}

Not applicable.

\section{Copyright}

(c) The Author(s) 2020.

\section{REFERENCES}

1. Siegel RL, Miller KD, Jemal A. Cancer statistics, 2020. CA Cancer J Clin 2020;70:7-30.

2. Govindan R, Page N, Morgensztern D, et al. Changing epidemiology of small-cell lung cancer in the United States over the last 30 years: analysis of the surveillance, epidemiologic, and end results database. J Clin Oncol 2006;24:4539-44.

3. Bunn PA Jr, Minna JD, Augustyn A, et al. Small cell lung cancer: can recent advances in biology and molecular biology be translated into improved outcomes? J Thorac Oncol 2016;11:453-74.

4. Nakazawa K, Kurishima K, Tamura T, et al. Specific organ metastases and survival in small cell lung cancer. Oncol Lett 2012;4:617-20.

5. Bernhardt EB, Jalal SI. Small cell lung cancer. In: Reckamp KL, editor. Lung Cancer. Cham: Springer International Publishing; 2016. pp. 301-22.

6. Horn L, Mansfield AS, Szczesna A, et al. First-line atezolizumab plus chemotherapy in extensive-stage small-cell lung cancer. $N$ Engl $J$ Med 2018;379:2220-9.

7. Paz-ares L, Dvorkin M, Chen Y, et al. Durvalumab plus platinum-etoposide versus platinum-etoposide in first-line treatment of extensivestage small-cell lung cancer (CASPIAN): a randomised, controlled, open-label, phase 3 trial. Lancet 2019;394:1929-39.

8. Waqar SN, Morgensztern D. Treatment advances in small cell lung cancer (SCLC). Pharmacol Ther 2017;180:16-23.

9. National Comprehensive Cancer Network. Small cell lung cancer. Version 3.2020. Available fron: https://www.nccn.org/professionals/ physician_gls/pdf/sclc.pdf [nccn.org]. [Last accessed on 04 Aug 2020 ].

10. Farago AF, Drapkin BJ, Lopez-Vilarino de Ramos JA, et al. ATLANTIS: a Phase III study of lurbinectedin/doxorubicin versus topotecan or cyclophosphamide/doxorubicin/vincristine in patients with small-cell lung cancer who have failed one prior platinum-containing line. Future Oncol 2019; 15:231-9.

11. Ardizzoni A, Hansen H, Dombernowsky P, et al: Topotecan, a new active drug in the second-line treatment of small-cell lung cancer: a phase II study in patients with refractory and sensitive disease. J Clin Oncol 1997;15:2090-6.

12. von Pawel J, Schiller JH, Shepherd FA, et al. Topotecan versus cyclophosphamide, doxorubicin, and vincristine for the treatment of recurrent small-cell lung cancer. J Clin Oncol 1999;17:658-67.

13. O'Brien ME, Ciuleanu TE, Tsekov H, et al. Phase III trial comparing supportive care alone with supportive care with oral topotecan in patients with relapsed small-cell lung cancer. J Clin Oncol 2006;24:5441-7.

14. Eckardt JR, von Pawel J, Pujol JL, et al. Phase III study of oral compared with intravenous topotecan as second-line therapy in small-cell lung cancer. J Clin Oncol 2007;25:2086-92.

15. Huber RM, Reck M, Gosse H, et al. Efficacy of a toxicity-adjusted topotecan therapy in recurrent small cell lung cancer. Eur Respir J 2006;27:1183-9.

16. Trigo J, Subbiah V, Besse B, et al. Lurbinectedin as second-line treatment for patients with small-cell lung cancer: a single-arm, openlabel, phase 2 basket trial. Lancet Oncol 2020;21:645-54.

17. Elez ME, Tabernero J, Geary D, et al. First-in-human phase I study of Lurbinectedin (PM01183) in patients with advanced solid tumors. Clin Cancer Res 2014;20:2205-14.

18. Calvo E, Moreno V, Flynn M, et al. Antitumor activity of lurbinectedin (PM01183) and doxorubicin in relapsed small-cell lung cancer: 
results from a phase I study. Ann Oncol 2017;28:2559-66.

19. Masuda N, Fukuoka M, Kusunoki Y, et al. CPT-11: a new derivative of camptothecin for the treatment of refractory or relapsed small-cell lung cancer. J Clin Oncol 1992;10:1225-9.

20. Fujita A, Takabatake H, Tagaki S, Sekine K. Pilot study of irinotecan in refractory small cell lung cancer. Gan To Kagaku Ryoho 1995;22:889-93.

21. Kondo R, Watanabe S, Shoji S, et al. A phase II study of irinotecan for patients with previously treated small-cell lung cancer. Oncology 2018;94:223-32.

22. Smit EF, Fokkema E, Biesma B, Groen HJ, Snoek W, Postmus PE. A phase II study of paclitaxel in heavily pretreated patients with smallcell lung cancer. Br J Cancer 1998;77:347-51.

23. Yamamoto N, Tsurutani J, Yoshimura N, et al. Phase II study of weekly paclitaxel for relapsed and refractory small cell lung cancer. Anticancer Res 2006;26:777-81.

24. Smyth J, Smith I, Sessa C, et al. Activity of docetaxel (Taxotere) in small cell lung cancer. Eur J Cancer 1994;30:1058-60.

25. Evans TL, Cho BC, Udud K, et al. Cabazitaxel versus topotecan in patients with small-cell lung cancer with progressive disease during or after first-line platinum-based chemotherapy. $J$ Thorac Oncol 2015;10:1221-8.

26. Pietanza MC, Kadota K, Huberman K, et al. Phase II trial of temozolomide in patients with relapsed sensitive or refractory small cell lung cancer, with assessment of methylguanine-DNA methyltransferase as a potential biomarker. Clin Cancer Res 2012;18:1138-45.

27. Pietanza MC, Waqar SN, Krug LM, et al. Randomized, double-blind, phase II study of temozolomide in combination with either veliparib or placebo in patients with relapsed-sensitive or refractory small-cell lung cancer. J Clin Oncol 2018;36:2386-94.

28. Zauderer MG, Drilon A, Kadota K, et al. Trial of a 5-day dosing regimen of temozolomide in patients with relapsed small cell lung cancers with assessment of methylguanine-DNA methyltransferase. Lung Cancer 2014;86:237-40.

29. Johnson DH, Greco FA, Strupp J, Hande KR, Hainsworth JD. Prolonged administration of oral etoposide in patients with relapsed or refractory small-cell lung cancer: a phase II trial. J Clin Oncol 1990;8:1613-7.

30. Einhorn LH, Pennington K, McClean J. Phase II trial of daily oral VP-16 in refractory small cell lung cancer: a Hoosier Oncology Group study. Semin Oncol 1990;17:32-35.

31. Jassem J, Karnicka-młodkowska H, van Pottelsberghe C, et al. Phase II study of vinorelbine (Navelbine) in previously treated small cell lung cancer patients. Eur J Cancer 1993;29:1720-2.

32. Drąg-Zalesińska M, Saczko J, Choromańska A, et al. Cisplatin and vinorelbine-mediated electrochemotherapeutic approach against multidrug resistant small cell lung cancer (H69AR) in vitro. Anticancer Res 2019;39:3711-8.

33. Schmittel A, Knödler M, Hortig P, Schulze K, Thiel E, Keilholz U. Phase II trial of second-line bendamustine chemotherapy in relapsed small cell lung cancer patients. Lung Cancer 2007;55:109-13.

34. Lammers PE, Shyr Y, Li CI, et al. Phase II study of bendamustine in relapsed chemotherapy sensitive or resistant small-cell lung cancer. $J$ Thorac Oncol 2014;9:559-62.

35. van der Lee I, Smit E, van Putten J, et al. Single-agent gemcitabine in patients with resistant small-cell lung cancer. Ann Oncol 2001;12:557-61.

36. Hoang T, Kim K, Jaslowski A, et al. Phase II study of second-line gemcitabine in sensitive or refractory small cell lung cancer. Lung Cancer 2003;42:97-102.

37. Yana T, Negoro S, Takada M, et al. Phase II study of amrubicin in previously untreated patients with extensive-disease small cell lung cancer: West Japan Thoracic Oncology Group (WJTOG) study. Invest New Drugs 2007;25:253-8.

38. von Pawel J, Jotte R, Spigel DR, et al. Randomized phase III trial of amrubicin versus topotecan as second-line treatment for patients with small-cell lung cancer. J Clin Oncol 2014;32:4012-9.

39. Spigel DR, Hainsworth JD, Lane CM, Clark B, Burris HA, Greco FA. Phase II trial of vinflunine in relapsed small cell lung cancer. $J$ Thorac Oncol 2010;5:874-8.

40. Gong J, Salgia R. Managing patients with relapsed small-cell lung cancer. J Oncol Pract 2018;14:359-66.

41. Hanna N, Bunn PA Jr, Langer C, et al. Randomized phase III trial comparing irinotecan/cisplatin with etoposide/cisplatin in patients with previously untreated extensive-stage disease small-cell lung cancer. J Clin Oncol 2006;24:2038-43.

42. Goto K, Ohe Y, Shibata T, et al. Combined chemotherapy with cisplatin, etoposide, and irinotecan versus topotecan alone as second-line treatment for patients with sensitive relapsed small-cell lung cancer (JCOG0605): a multicentre, open-label, randomised phase 3 trial. Lancet Oncol 2016;17:1147-57.

43. Antonia SJ, López-martin JA, Bendell J, et al. Nivolumab alone and nivolumab plus ipilimumab in recurrent small-cell lung cancer (CheckMate 032): a multicentre, open-label, phase 1/2 trial. Lancet Oncol 2016;17:883-95.

44. Ready NE, Ott PA, Hellmann MD, et al. Nivolumab monotherapy and nivolumab plus ipilimumab in recurrent small cell lung cancer: results from the CheckMate 032 Randomized Cohort. J Thorac Oncol 2020;15:426-35.

45. Reck M, Vicente D, Ciuleanu T, et al. Efficacy and safety of nivolumab (nivo) monotherapy versus chemotherapy (chemo) in recurrent small cell lung cancer (SCLC): results from CheckMate 331. Ann Oncol 2018;29:x43.

46. Ott PA, Elez E, Hiret S, et al. Pembrolizumab in patients with extensive-stage small-cell lung cancer: results from the phase Ib KEYNOTE-028 study. J Clin Oncol 2017;35:3823-9.

47. Chung HC, Piha-Paul SA, Lopez-Martin, et al. Phase 2 study of pembrolizumab in advanced small-cell lung cancer (SCLC): KEYNOTE-158. J Clin Oncol 2018;36:Abstract 8506

48. Chung HC, Piha-Paul SA, Lopez-Martin J, et al. Pembrolizumab after two or more lines of previous therapy in patients with recurrent or metastatic SCLC: results from the KEYNOTE-028 and KEYNOTE-158 studies. $J$ Thorac Oncol 2020;15:618-27. 
49. Cho DC, Mahipal A, Dowlati A, et al. Safety and clinical activity of durvalumab in combination with tremelimumab in extensive disease small-cell lung cancer (ED-SCLC). J Clin Oncol 2018;36:8517.

50. Owonikoko T, Higgins $\mathrm{K}$, Chen ZJ, et al. A randomized phase II study of tremelimumab and durvalumab with or without radiation for patients with relapsed small cell lung cancer (SCLC). J Clin Oncol 2019;37:8515.

51. Chiang AC, Sequist LVD, Gilbert J, et al. Clinical activity and safety of Atezolizumab in a phase 1 study of patients with relapsed/ refractory small-cell lung cancer. Clin Lung Cancer 2020;21:455-63.

52. Pujol JL, Lavole A, Quoix E, et al. Randomized phase II-III study of bevacizumab in combination with chemotherapy in previously untreated extensive small-cell lung cancer: results from the IFCT-0802 trial. Ann Oncol 2015;26:908-14.

53. Arnold AM, Seymour L, Smylie M, et al. Phase II study of vandetanib or placebo in small-cell lung cancer patients after complete or partial response to induction chemotherapy with or without radiation therapy: National Cancer Institute of Canada Clinical Trials Group Study BR.20. J Clin Oncol 2007;25:4278-84.

54. Allen JW, Moon J, Redman M, et al. Southwest Oncology Group S0802: a randomized, phase II trial of weekly topotecan with and without ziv-aflibercept in patients with platinum-treated small-cell lung cancer. J Clin Oncol 2014;32:2463-70.

55. Belani CP, Dahlberg SE, Rudin CM, et al. Vismodegib or cixutumumab in combination with standard chemotherapy for patients with extensive-stage small cell lung cancer: a trial of the ECOG-ACRIN Cancer Research Group (E1508). Cancer 2016;122:2371-8.

56. de Marinis F, Atmaca A, Tiseo M, et al. A phase II study of the histone deacetylase inhibitor panobinostat (LBH589) in pretreated patients with small-cell lung cancer. $J$ Thorac Oncol 2013;8:1091-4.

57. Rudin CM, Salgia R, Wang X, et al. Randomized phase II Study of carboplatin and etoposide with or without the bcl-2 antisense oligonucleotide oblimersen for extensive-stage small-cell lung cancer: CALGB 30103. J Clin Oncol 2008;26:870-6.

58. Langer CJ, Albert I, Ross HJ, et al; GEM017 Investigators. Randomized phase II study of carboplatin and etoposide with or without obatoclax mesylate in extensive-stage small cell lung cancer. Lung Cancer 2014;85:420-8.

59. Melichar B, Adenis A, Lockhart AC, et al. Safety and activity of alisertib, an investigational aurora kinase A inhibitor, in patients with breast cancer, small-cell lung cancer, non-small-cell lung cancer, head and neck squamous-cell carcinoma, and gastro-oesophageal adenocarcinoma: a five-arm phase 2 study. Lancet Oncol 2015;16:395-405.

60. Yang S, Zhang Z, Wang QM. Emerging therapies for small cell lung cancer. J Hematol Oncol 2019;12:47.

61. Koinis F, Agelaki S, Karavassilis V, et al. Second-line pazopanib in patients with relapsed and refractory small-cell lung cancer: a multicentre phase II study of the Hellenic Oncology Research Group. Br J Cancer 2017;117:8-14.

62. Cheng Y, Wang Q, Li K, et al. OA13.03 anlotinib as third-line or further-line treatment in relapsed SCLC: a multicentre, randomized, double-blind phase 2 trial. $J$ Thorac Oncol 2018;13:S351-2.

63. Cheng Y, Wang Q, Li K, et al. Overall survival (OS) update in ALTER 1202: Anlotinib as third-line or further-line treatment in relapsed small-cell lung cancer (SCLC). Ann Oncol 2019;30:v710-7.

64. Rogado J, Pangua C, Serrano-Montero G, et al. Covid-19 and lung cancer: a greater fatality rate? Lung Cancer 2020;146:19-22. 